\title{
Diagnóstico, factores críticos y lecciones de aprendizaje de proyectos fondo emprender en el Huila 2002-2014
}

\author{
Diagnosis, critical factors and learning lessons \\ of Fondo Emprender projects in Huila 2002-2014
}

\author{
Rafael Armando Méndez Lozano \\ Derly Cibelly Lara Figueroa \\ Universidad Surcolombiana
}

\begin{abstract}
Resumen
La sociedad muestra una tendencia hacia las organizaciones como respuesta a las necesidades humanas; no obstante, la comprensión del proceso de creación de empresas continúa siendo muy limitada, a pesar de múltiples planteamientos que permiten destacar tres corrientes de pensamiento: la Escuela Shumpeteriana, la Escuela Austriaca y la Teoría de la Acción Planeada. Hoy se reconoce que los investigadores aún están lejos de alcanzar un consenso sobre las características del mundo empresarial y es posible desde el estudio de la realidad empresarial, identificar factores críticos y lecciones de aprendizaje para mejorar las prácticas en los procesos de capacitación, asesoría, acompañamiento y gestión en el desarrollo de iniciativas emprendedoras a partir del estudio de experiencias de proyectos financiados con recursos del Fondo Emprender en el departamento del Huila.
\end{abstract}

Para abordar el estudio se tuvieron en cuenta los postulados de la Teoría de la Acción, siguiendo a Gartner (1985) y (1989), Bolaños (2006), con base en los análisis de Cardona, Dinora y Tabares (2012) que permiten relacionar la acción de emprender, como el resultado de la combinación de factores internos del individuo $\mathrm{y}$ factores externos en tres niveles económicos macro, meso y micro que determinan la creación de empresas como un proceso multidimensional en el que influye en cada contexto el individuo, el entorno, el proceso y la organización.

El marco epistemológico desde el que se aborda el estudio investigativo es de orden Interpretativista, desde una metodología cualitativa, con la intención de explorar, comprender, interpretar y explicar el fenómeno concentrado en las fortalezas y debilidades de las iniciativas empresariales desarrolladas por los emprendedores (empresarios) que han participado en las convocatorias del Fondo Emprender, así como de actores secundarios de gran incidencia en el proceso de la cadena de valor desde la idea hasta la constitución de la empresa y se complementa con la

\section{Abstract}

Society shows a tendency towards organizations as the answer to human needs; nevertheless, understanding of the process of creating companies continues to be very limited, in spite of multiple approaches that allow us to highlight three schools of thought: The Schumpeterian School, the Austrian School and the Planned Action Theory. Today it is recognized that researchers are still far from reaching a consensus on the characteristics of the business world and it is possible, from the study of the business reality, to identify critical factors and learned lessons to improve practices in the training, consulting, accompaniment, and management processes in the development of entrepreneur initiatives starting from the study of experiences of projects financed by resources of Fondo Emprender in the Department of Huila.

The principles of the Action Theory were considered to approach the study, following Gardner (1985) and (1989), Bolaños (2006), based on the analyses of Cardona, Dinora and Tabares (2012) that allow the relation of the action of undertaking, as the result of the combination of internal factors of the individual and external factors in three economic levels macro, meso and micro that determine the creation of companies as a multidimensional process in which in each context the individual, the environment, the process and the organization influence.

The epistemological framework from which the research study is approached is of an interpretative order, form a qualitative methodology, with the intention of exploring, understanding, interpreting, and explaining the phenomenon concentrated on the strengths and weaknesses of business initiatives developed by entrepreneurs (businessmen) that have participated in the calls of Fondo Emprender, as well as secondary actors of great incidence in the process of the value chain from the idea to the incorporation of the Company and it is complemented with consulting reliable sources of secondary information. 
consulta a fuentes confiables de información secundaria.

De acuerdo al plan de trabajo, los actores identificados y las categorías de individuos seleccionados para la aplicación de los instrumentos elaborados con base en el marco teórico seleccionado y la revisión documental realizada por los investigadores, se procedió a efectuar diecisiete entrevistas en total, las cuales fueron procesadas y analizadas con base en el software Atlas $\mathrm{Ti}$, el cual permitió inductivamente desarrollar el analisis descriptivo y la consideración de nuevos codigos emergentes necesarios para dar respuesta al proposito del estudio en función del individuo, la organización y el proceso, el ciclo del proyecto (pre-inversión, inversión y funcionamiento) y el entorno. Las lecciones de aprendizaje resultado de los trabajos realizados, permiten caracterizar las experiencias de los proyectos financiados por en Fondo Emprender en el Huila, aportar nuevas lecturas desde un enfoque cualitativo e identificar temas para futuras investigaciones.

Palabras clave: Emprendimiento, Competitividad, Innovación, Empresa, Entorno, Región
According to the work plan, the actors identified and the categories of the selected individuals for the application of the instruments elaborated based on the theoretical framework chosen and the documentary review carried out by the researchers, it was proceeded to carry out seventeen interviews in all, which were processed and analyzed based on the Atlas Ti software, which allowed to inductively develop the descriptive analysis and the consideration of new emerging codes necessary to give a response to the purpose of the study in terms of the individual, the organization and the process, the cycle of the project (pre-investment, investment and functioning) and the surroundings. The learned lessons resulting form the work done, allow the characterization of the experiences of the projects financed by Fondo Emprender in Huila, provide new readings from a qualitative approach and to identify topics for future research.

Key Words: Entrepreneurship, competitiveness, innovation, business, surroundings, region

\section{Introducción}

Calificado como un campo de conocimiento en construcción, disciplina joven y emergente (Orrego, 2012) el Emprendimiento, inscrito en la Disciplina de la Administración, se encuentra en proceso de cohesión y solidez, por ello asume la condición, actual, como campo pre científico.

El fenómeno del Emprendimiento, siguiendo a Formichella (2004), puede definirse "como el desarrollo de un proyecto que persigue un determinado fin económico, político o social, entre otros, y que posee ciertas características, principalmente que tiene una cuota de incertidumbre y de innovación" (p. 3). Ser emprendedor, significa estar en la capacidad de crear algo nuevo o de dar un nuevo uso a algo existente, en perspectiva por generar un impacto a nivel personal y en la comunidad en la que el sujeto se encuentra.

El origen y definición del término, Emprendedor, tiene incidencia por primera vez inicialmente en la literatura económica, en los inicios del siglo XVIII por el economista Francés Richard Chantillón, con la palabra Entrepreneur. Cantillo define Entrepreneur -emprendedor- como "agente que compra los medios de producción a ciertos precios y los combina en forma ordenada para obtener de allí un nuevo producto" (Formichella 2004, p10), agente caracterizado porque no posee un retorno seguro, afirmando que él es quien, soporta y asume los riesgos que proporciona el comportamiento del mercado (Formichella, 2004). Burnet (2000), citado por (Formichella, 2004), afirma que es el economista francés Say, quien hace un interesante aporte a la definición de Cantillon. Say afirma que el entrepreneur es un individuo líder, previsor, tomador de riesgos y evaluador de proyectos, que moviliza recursos desde una zona de bajo rendimiento a una de alta productividad (Formichella, 2004). En 1880 Alfred Marshall introduce la necesidad del entrepreneurship para la producción, al afirmar que los factores de producción no son tres, sino cuatro: Tierras, trabajo, capital y Organización -factor coordinador de las otras tres-. En ese sentido, para Marshall, el entreprenurship es el elemento que está detrás de la organización, manejándola, estableciendo que los emprendedores son líderes por naturaleza, dispuestos a actuar bajos las condiciones de incertidumbre que causa la información asimétrica (Formichella, 2004). Joseph Schumpeter (1911), retoma el concepto de Say, en su libro "Teoría de la dinámica económica", plantea la existencia del desequilibrio dinámico, causado por el emprendedor, quienes realizan las tareas de "destrucción creativa" (Formichella 2004, p11). Para Schumpeter (1942) el término emprendedor se refiere aquellos individuos que con sus acciones causan inestabilidades en los mercados. Drucker (1985), reconocido por su aportes a la teoría del emprendimiento, define al entrepreneur, siguiendo a (Formichella, 2004), como "aquel empresario que es innovador (y al entrepreneurship como el empresariado innovador), y aclara la común confusión de creer que cualquier negocio pequeño y nuevo es un emprendimiento, y quien lo lleva a cabo es un emprendedor" (p.13).

Howard Stevenson, en la década de los 80, profesor de la Universidad de Harvard, en un análisis acerca de la mentalidad emprendedora, concluye siguiendo a Castillo (1999) citado por (Formichella, 2004), que está se basa más en las oportunidades que en los recursos. 
Para Stevenson, crear una empresa no es suficiente para ser considerado emprendedor. Un emprendedor, además de ser el creador de una organización, debe buscar continuamente la oportunidad y crear riqueza, innovando, como eje central del desarrollo de su emprendimiento.

\section{Emprendimiento Empresarial}

En el marco del estudio investigativo sobre el Emprendimiento, se destacan tres corrientes de pensamiento: la escuela Schumpeteriana, la Escuela Austriaca y la Teoría de la Acción Planeada.

Cuadro 1. Escuela Schumpeteriana, Austriaca, Teoría de la acción planeada

\begin{tabular}{|c|c|c|}
\hline $\begin{array}{c}\text { Escuela } \\
\text { Schumpeteriana }\end{array}$ & Escuela Austriaca & $\begin{array}{l}\text { Teoría de la acción } \\
\text { planeada } \\
\text { Bird, 1988; Boyd y } \\
\text { Vozikis,1994; } \\
\text { Crant,1996; Katz y } \\
\text { Gartner, Shapero } \\
\text { (1982), entre otros }\end{array}$ \\
\hline $\begin{array}{l}\text { *Promotor de nuevas } \\
\text { combinaciones o } \\
\text { innovaciones. } \\
\text { *Reformar o revolucionar } \\
\text { el patrón de producción } \\
\text { al explotar una invención } \\
\text { para: } \\
\text { a) Producir un nuevo } \\
\text { producto } \\
\text { b) Proveer de una nueva } \\
\text { fuente de i nsumos o un } \\
\text { material nuevo } \\
\text { c) Reorganizar una } \\
\text { industria, etc. } \\
\text { *Además de las } \\
\text { capacidades técnicas y } \\
\text { la experiencia, el } \\
\text { ejercicio de la intuición y } \\
\text { la estrategia es de } \\
\text { particular importancia en } \\
\text { el proceso emprendedor. }\end{array}$ & $\begin{array}{l}\text { *Especular en una } \\
\text { situación de } \\
\text { incertidumbre, responde a } \\
\text { las señales del mercado } \\
\text { con respecto a precios, } \\
\text { ganancias y pérdidas. } \\
\text { *Ayuda a nivelar la } \\
\text { demanda y la oferta y, si } \\
\text { ha especulado } \\
\text { correctamente. } \\
\text { *Generara una ganancia } \\
\text { para sí, asume las } \\
\text { perdidas por su decisión } \\
\text { incorrecta } \\
\text { *El factor emprendedor } \\
\text { está presente en todas las } \\
\text { acciones humanas debido } \\
\text { a las incertidumbres del } \\
\text { contexto. } \\
\text { *No limitado a las } \\
\text { acciones de un tipo } \\
\text { particular de personas. }\end{array}$ & $\begin{array}{l}\text { *El comportamiento } \\
\text { emprendedor es } \\
\text { pronosticado a partir } \\
\text { de la intención. } \\
\text { *La intención se } \\
\text { identifica a partir de } \\
\text { tres variables: } \\
\text { a) El control interno } \\
\text { b) El comportamiento } \\
\text { planificado } \\
\text { c) La intencionalidad } \\
\text { *La aplicación del } \\
\text { modelo de la acción } \\
\text { planeada se propone } \\
\text { de manera similar al } \\
\text { modelo de las } \\
\text { intensiones donde la } \\
\text { acción de emprender } \\
\text { es vista como } \\
\text { deseable y viable por } \\
\text { el emprendedor. } \\
\text { *Estudia las influencias } \\
\text { de las redes sociales a } \\
\text { las que pertenece el } \\
\text { individuo sobre su } \\
\text { comportamiento } \\
\text { (ventura: } 2006: 7 \text { ) }\end{array}$ \\
\hline
\end{tabular}

Fuente: Elaborado por Cardona, Vera \& Tabares (2008) basado en Carrillo: 2006, Ventura: 2006 y Kirzner: 1998. En las dimensiones del emprendimiento empresarial: La experiencia de los programas Cultura Ey Fondo Emprender en Medellín (Pág. 15)

Para el presente estudio se tomarán como base los postulados de la Teoría de la Acción, desde los estudios del comportamiento, siguiendo el trabajo de Gartner (1985) y (1989) el cual fue sintetizado y estudiado por Cardona, Dinora, y Tabares (2012), en su desarrollo investigativo "En las dimensiones del emprendimiento empresarial: La experiencia de los programas Cultura E y Fondo Emprender en Medellín". En ese sentido, comprendiendo la pertinencia de este enfoque, las bases o preceptos que permiten adentrarnos al entramado teórico propio para el presente estudio, se asume que:

1) La intención del individuo es el factor clave para comprender el proceso del emprendimiento.

2) Se reconoce al individuo como evaluador de las condiciones requeridas para emprender (condiciones tanto internas como externas). 
3) Manifiesta la importancia de las redes sociales para desarrollar la actividad emprendedora.

4) Tienen en cuenta otras dimensiones que se relacionan entre si y que son cruciales para el desarrollo emprendedor y empresarial. (Cardona et al. 2012, p15)

Resulta esencial entender al emprendimiento desde una perspectiva empresarial, conocido entonces como emprendimiento empresarial. Para Gartner (1989), siguiendo a (Cardona et al. 2012), el emprendimiento es un proceso de creación de empresas e implica tener en cuenta dos aspectos:

- Las organizaciones son creadas por gente que no es emprendedora (partidos políticos, asociaciones y grupos sociales

Cuadro 2. Características Emprendedoras y Empresario
- Evaluar la capacidad de acción como emprendedor, no hay rasgos que indiquen una forma específica para definir criterios de emprendimientos. Reconoce el autor, siguiendo a Cardona et al. (2012) que el emprendedor es aspecto intrínseco para el emprendimiento. (p.15)

En el propósito de lograr sumergirnos en el conocimiento y caracterización de los empresarios emprendedores financiados por el Fondo Emprender, los procesos relacionados con el desarrollo de su emprendimiento y la creación de organizaciones empresariales, se toman los elementos configurados por Bolaños (2006) y Gartner (1985:1989), quienes han coincidido siguiendo el estudio desarrollado por Cardona et al. (2012) en enunciar importantes características y factores que abordan al individuo a nivel interno y externo.

\begin{tabular}{|c|c|c|}
\hline & Emprendedor & Empresario \\
\hline Individuo & $\begin{array}{l}\text { Pionero } \\
\text { Incertidumbre } \\
\text { Crear algo nuevo } \\
\text { Adapta ideas } \\
\text { Creativo } \\
\text { Autónomo } \\
\text { Tiene confianza en si mismo } \\
\text { Desarrolla las oportunidades } \\
\text { Toma de riesgo } \\
\text { Esfuerzo por sobresalir } \\
\text { Flexible Dinámico }\end{array}$ & $\begin{array}{l}\text { Necesidad de logro } \\
\text { Cree en sus capacidades } \\
\text { Cumple metas y objetivos } \\
\text { Es innovador } \\
\text { Crea valor } \\
\text { Impulsa la creación de una } \\
\text { organización, busca el beneficio } \\
\text { Toma decisiones } \\
\text { Busca el crecimiento } \\
\text { Impulsor perspicaz }\end{array}$ \\
\hline Entorno & $\begin{array}{l}\text { Identificación de oportunidades } \\
\text { Evaluación y elección de estas } \\
\text { oportunidades } \\
\text { Previsor } \\
\text { Manejo inestabilidad en los } \\
\text { mercados } \\
\text { Dinamiza la industria } \\
\text { Investiga }\end{array}$ & $\begin{array}{l}\text { Control y responsabilidad } \\
\text { Conocimiento del contexto } \\
\text { Sostenibilidad } \\
\text { Competitividad } \\
\text { Manejo de recursos, productos y } \\
\text { equipos de trabajo } \\
\text { Manejo de información } \\
\text { Manejo de fondos financieros y } \\
\text { planes de negocio } \\
\text { Capacidad de gestión }\end{array}$ \\
\hline Proceso & $\begin{array}{l}\text { Relación con amigos, familia } \\
\text { Instituciones de apoyo financiero } \\
\text { y formativo }\end{array}$ & $\begin{array}{l}\text { Establecimiento de redes en } \\
\text { cadenas productivas } \\
\text { Reconocimiento en el medio } \\
\text { para generar sostenibilidad } \\
\text { Identifica características de las } \\
\text { personas con las cuales trabaja, } \\
\text { se proyecta. }\end{array}$ \\
\hline
\end{tabular}

Fuente: Elaborado por Cardona et al. (2008) basado en Gartner (1989) y Bolaños 2006. En las dimensiones del emprendimiento empresarial: La experiencia de los programas Cultura Ey Fondo Emprender en Medellín (Pág. 18) 
Para Bolaños (2006), de acuerdo con Cardona et al., (2012) la decisión de emprender es un proceso propio interno- que el emprendedor realiza, el cual se ve influenciado tanto por factores internos como externos, agrupados estos, en tres niveles económicos: macro -factores como las regulaciones, impuestos, oportunidades y tratados de libre comercio que pueden influir-, meso -el tejido empresarial y la tendencia a la asociatividad de la región que también juega un papel fundamental- y micro - la facilidad de organizar la empresa y la facilidad de encontrar mano de obra y empleados competentes es vital-.
Bolaños (2006) hace referencia dentro de los factores internos a elementos adquiridos -propios del individuo y el entorno-, destacando dentro de los factores internos que estos no solo hacen referencia a "la actitud del individuo con base en factores hereditarios sino que tienen que ver con la forma como asume su relación con el entorno." (Cardona et al. 2008, p.17). Por su parte Gartner (1985) plantea la creación de empresas como un fenómeno multidimensional, compuesto por cuatro dimensiones -individual, entorno, proceso y organización-, en el cual se debe considerar el grado de complejidad de la interacción de las variables.

Cuadro 3. Factores y Dimensiones del emprendimiento empresarial -Bolaños (2006) y Gartner (1985:1989)

\begin{tabular}{|c|c|c|c|}
\hline Factores (Bolaños) & \multicolumn{3}{|c|}{ Dimensiones (Gartner) } \\
\hline $\begin{array}{l}\text { Internos } \\
\text { Variables del } \\
\text { individuo que } \\
\text { determinan el }\end{array}$ & \multicolumn{3}{|c|}{$\begin{array}{l}\text { Necesidad de logro, control de locus, propensión a toma } \\
\text { de riesgo, experiencia, satisfacción del trabajo, p adres } \\
\text { empresarios, edad, educación. }\end{array}$} \\
\hline $\begin{array}{l}\text { Externos } \\
\text { Estado, las leyes, los } \\
\text { tratados comerciales, } \\
\text { incentivos fiscales, } \\
\text { esquemas de } \\
\text { financiamiento, } \\
\text { recursos } \\
\text { tecnológicos, } \\
\text { humanos y } \\
\text { tecnológicos. }\end{array}$ & $\begin{array}{l}\text { Entorno } \\
\text { Capital } \\
\text { disponible } \\
\text { Acceso a } \\
\text { proveedores } \\
\text { Consumidores } \\
\text { Transporte } \\
\text { Recursos } \\
\text { Financieros } \\
\text { Instalaciones } \\
\text { Habilidades } \\
\text { técnicas } \\
\text { Entornos } \\
\text { Condiciones } \\
\text { urbanas, de } \\
\text { vida etc. }\end{array}$ & $\begin{array}{l}\text { Proceso } \\
\text { Oportunidades de } \\
\text { negocio } \\
\text { Los empresarios } \\
\text { El mercado } \\
\text { Mercado de } \\
\text { productos y } \\
\text { servicios } \\
\text { Construcción de } \\
\text { redes }\end{array}$ & $\begin{array}{l}\text { Organización } \\
\text { Diferenciación } \\
\text { Innovación } \\
\text { Cambio de } \\
\text { regulación } \\
\text { Competencias } \\
\text { paralelas } \\
\text { Transferencias }\end{array}$ \\
\hline
\end{tabular}

Fuente: Las Dimensiones del emprendimiento empresarial: La experiencia de los programas Cultura E y Fondo Emprender en Medellín (Cardona et al. 2008) (P. 17)

Siguiendo el trabajo Gartner (1985:1989), se presenta en el esquema 3 las variables que tanto el individuo como el entorno deben desarrollar, en aras por generar un escenario posible para la creación de empresas. Las "Dimensiones de creación de nuevas empresas" presentado por (Cardona, Dinora \& Tabares, 2012) incluye variables, siguiendo a las investigadoras por los autores McClelland (1961) y Timmons (1999) en Varela (2001:7475) que se enmarcan dentro de este mismo esquema de análisis.

En ese sentido, y ante la necesidad de conocer la experiencia regional y plantear mejoras metodológicas en el proceso de asesoría, acompañamiento y desarrollo de iniciativas de los emprendedores, se pretende con la investigación desde una perspectiva fundamentalmente cualitativa, cconocer el estado actual, los factores 
críticos y las lecciones de mejoramiento de las iniciativas empresariales financiadas con el Fondo Emprender en el Huila en el periodo comprendido entre 2002 y 2013.

\section{Metodología}

El marco epistemológico desde el que se abordó el presente estudio investigativo es de orden Interpretativista, desde una metodología cualitativa, con la intención de explorar, comprender, interpretar y explicar el fenómeno concentrado en las fortalezas $y$ debilidades de las iniciativas empresariales desarrolladas por emprendedores (empresarios) en el departamento del Huila financiados por el Fondo Emprender -programa de financiamiento empresarial-, considerando además, el proceso que se vivencia en la búsqueda para lograr financiar y desarrollar su propuesta de proyecto productivo.

El presente estudio se fundamentó en la triangulación de fuentes, es decir, desde la "Triangulación de fuentes o recogidas de información de diversa procedencia." (Ruiz, 1999). Para el caso de estudio la información fue tomada esencialmente desde emprendedores (empresarios) participantes junto a actores secundarios de gran incidencia en el proceso de la cadena de valor desde la idea hasta la constitución de la empresa.

\section{Fuentes para la recolección de la información}

La investigación centró los esfuerzos en el conocimiento e inmersión en primera medida en los emprendedores (Empresarios), caracterizados por haber participado en las convocatorias regionales o nacionales del Fondo Emprender.

Fue esencial en el proceso de desarrollo del presente estudio, caracterizar los "tipos de emprendedores y empresarios" participantes. De acuerdo a la normatividad establecida por el Fondo Emprender, en el artículo 12 numeral 1 del acuerdo 004 del 2009, los emprendedores solo podrán ser beneficiados con los recursos del Fondo Emprender por una sola vez, en tanto podrán concursar las veces que estén dispuestos a hacerlo en las convocatorias abiertas.

En ese orden de ideas, fue posible reconocer tres situaciones que permitieron caracterizar a los emprendedores participantes en el estudio:

- Emprendedor(es) que lograron constituirse como empresario(s) y su empresa se encuentra en el primer año de operación o funcionamiento y es objeto de interventoría.

- Empresario(s) que cumplieron sus metas durante los años de seguimiento y fueron beneficiados con la condonación del capital semilla asignado y continua(n) en funcionamiento -Emprendimiento exitoso-.
- Empresarios para quien(es) su emprendimiento a pesar de haber sido favorecido por el Fondo Emprender, 1) decidieron no recibir el capital semilla, 2) o recibieron los aportes del Fondo Emprender, pero no cumplieron con las metas pre-establecidas no fueron beneficiados de la condonación del capital semilla y/o 3) aquellos que a pesar de haber recibido la condonación de la deuda, su iniciativa empresarial fracasó posteriormente y el emprendimiento no está en operación

Los actores secundarios del estudio corresponden a los representantes e integrantes de las siguientes organizaciones:

- Fondo Financiero de Proyectos de Desarrollo FONADE-

- Fondo de Financiamiento Empresarial -Fondo Emprender- a nivel nacional y regional.

- Líderes de las Unidades de Emprendimiento -SENAde los cuatro municipios representativos del departamento del Huila: Neiva, Garzón, Pitalito y La Plata.

- Ex participantes e integrantes de las instituciones coordinadoras -FONADE y Fondo Emprender.

Como fuentes secundarias se consultaron:

- Informes de Gestión FONADE y SENA -Fondo Emprender-2002-2014

- Estadísticas a nivel nacional y regional relacionada con los proyectos presentados 2002-2014.

- Páginas web FONADE y Fondo Emprender

- Normatividad Jurídica Fondo Emprender y FONADE

- Artículos periodísticos y artículos investigativos relacionados con la temática de interés.

Considerando los objetivos planteados, las técnicas más apropiadas para la recolección la información fueron:

- La Entrevista exploratoria, semidirigida, estructurada

- Análisis de contenido

- La Observación directa

\section{Análisis}

De forma sistemática, coherente y rigurosa, el análisis de los datos cualitativos para el presente estudio se realizó en varias fases. Los objetivos -generales y específicos- permitieron establecer como punto de partida, un trabajo preliminar de entrevistas exploratorias, que permitieron una aproximación a la ruta de trabajos de campo y hacer una revisión desde la perspectiva teórica en relación con las temáticas de la investigación, así como plantear metodológicamente la selección y el uso de los instrumentos para la recolección de los datos necesarios.

En la perspectiva del plan de trabajo, los actores 
identificados y las categorías de individuos seleccionados para la aplicación de los instrumentos elaborados con base en el marco teórico seleccionado y la revisión documental realizada por los investigadores, se efectuaron diecisiete entrevistas en total, pertenecientes a las siguientes categorías:

Figura 1. Actores entrevistados

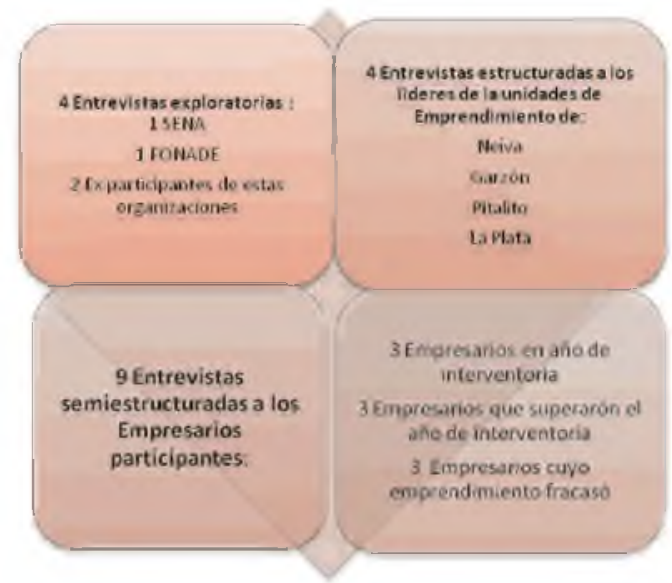

Fuente: Elaboración propia
El procesamiento para el análisis de los datos cualitativos se llevó a cabo utilizando el software Atlas $\mathrm{Ti}$, el cual facilitó la definición y manejo de las categorías. Las citas bibliográficas y aspectos relevantes derivados del procesamiento se ordenaron en una matriz Excel denominada "categorías teóricas u emergentes", logrando así organizar, reagrupar y gestionar el material de manera rigurosa.

Para auscultar la información obtenida, se realizó un análisis de contenido doble caracterizado por ser Manifiesto, mediante el cual "el investigador busca palabras específicas" y Latente, en cuanto "busca el significado de palabras especificas en el contexto de todos los datos" y así se "determina una categoría apropiada" (Mayan, 2001, p.22). En ese orden de ideas, las categorías teóricas preestablecidas fueron estudiadas teniendo como referencia el análisis Manifiesto y se crearon categorías emergentes denominadas "Factores críticos", las cuales fueron estudiadas para cada perfil y se examinaron elementos positivos y negativos dentro del proceso experimentado en la iniciativa emprendedora, así como en el ser y vivencia de los emprendedores.

Tabla 1. Categorías recurrentes en el estudio

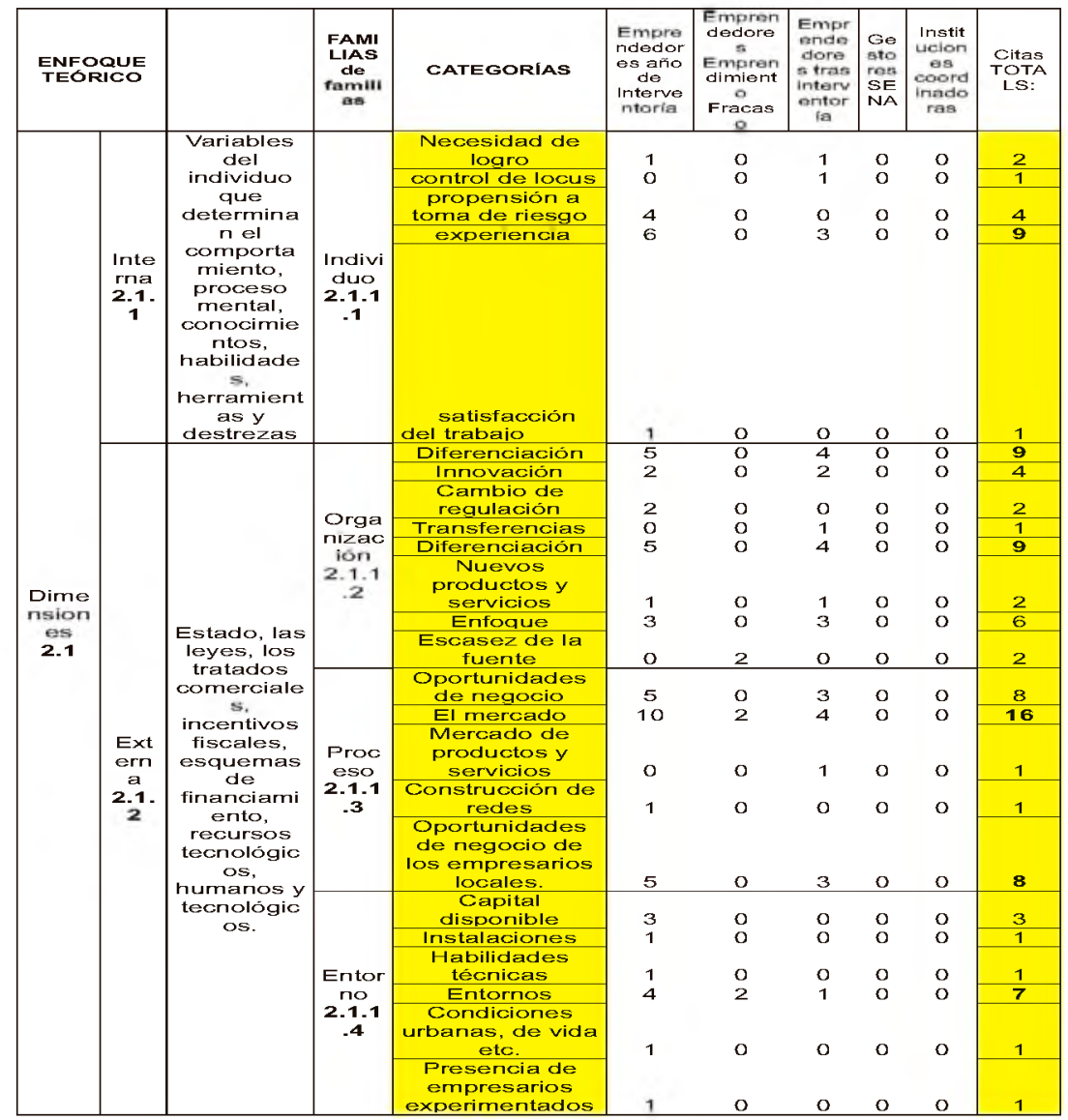

Fuente: Elaboración Propia -Analisis Manifiesto- 
Tabla 2. Categorias recurrentes en el estudio

\begin{tabular}{|c|c|c|c|c|c|c|c|c|c|}
\hline $\begin{array}{l}\text { ENFOQUE } \\
\text { TEORICO }\end{array}$ & $\begin{array}{l}\text { Famili } \\
\text { as }\end{array}$ & $\begin{array}{c}\text { Famil } \\
\text { IAS } \\
\text { de } \\
\text { familia } \\
\text { s }\end{array}$ & CATEGORIAS & $\begin{array}{c}\text { Em } \\
\text { pren } \\
\text { ded } \\
\text { ores } \\
\text { año } \\
\text { de } \\
\text { Inter } \\
\text { vent } \\
\text { oría }\end{array}$ & $\begin{array}{c}\text { Em } \\
\text { pren } \\
\text { ded } \\
\text { ores } \\
\text { Em } \\
\text { Pren } \\
\text { dimi } \\
\text { onto } \\
\text { Frao } \\
\text { aso }\end{array}$ & $\begin{array}{l}\text { Em } \\
\text { pre } \\
\text { nd } \\
\text { ed } \\
\text { ore } \\
\text { s } \\
\text { tra } \\
\text { s } \\
\text { int } \\
\text { erv } \\
\text { ent } \\
\text { orí }\end{array}$ & $\begin{array}{l}\text { Ge } \\
\text { sto } \\
\text { res } \\
\text { SE } \\
\text { NA }\end{array}$ & $\begin{array}{l}\text { Ins } \\
\text { titu } \\
\text { cio } \\
\text { ne } \\
\text { s } \\
\text { co } \\
\text { ord } \\
\text { ina } \\
\text { dor } \\
\text { as }\end{array}$ & $\begin{array}{l}\text { T } \\
\text { Oे } \\
\text { ' } \\
\text { A } \\
\text { L } \\
\text { S: }\end{array}$ \\
\hline \multirow{21}{*}{$\begin{array}{c}\text { Bolaños } \\
\text { (2006)y } \\
\text { Gartnet } \\
\text { característi } \\
\text { cas y } \\
\text { factores } \\
\text { que } \\
\text { abordan al } \\
\text { individuo a } \\
\text { nivel } \\
\text { intornoy } \\
\text { externo- }\end{array}$} & \multirow{9}{*}{$\begin{array}{l}\text { Empre } \\
\text { ndedor }\end{array}$} & \multirow{5}{*}{$\begin{array}{l}\text { Individ } \\
\text { wo }\end{array}$} & $\begin{array}{l}\text { Incertidumbre } \\
\text { Adapta ideas } \\
\text { Creativo } \\
\text { Autónomo }\end{array}$ & $\begin{array}{l}2 \\
1 \\
2 \\
4\end{array}$ & $\begin{array}{l}0 \\
0 \\
0 \\
0\end{array}$ & $\begin{array}{l}1 \\
1 \\
1 \\
1\end{array}$ & $\begin{array}{l}0 \\
0 \\
0 \\
0\end{array}$ & $\begin{array}{l}0 \\
0 \\
0 \\
0\end{array}$ & $\begin{array}{l}3 \\
2 \\
3 \\
5\end{array}$ \\
\hline & & & $\begin{array}{l}\text { Tiene confianza en si } \\
\text { mismo }\end{array}$ & \multirow{4}{*}{$\begin{array}{l}4 \\
4 \\
6 \\
4 \\
4 \\
0\end{array}$} & o & \multirow{4}{*}{$\begin{array}{l}2 \\
0 \\
2 \\
2 \\
1\end{array}$} & \multirow{4}{*}{$\begin{array}{l}0 \\
0 \\
0 \\
0 \\
0\end{array}$} & \multirow{4}{*}{$\begin{array}{l}0 \\
0 \\
0 \\
0 \\
0\end{array}$} & 6 \\
\hline & & & $\begin{array}{l}\text { Desarrolla las } \\
\text { oportunidades }\end{array}$ & & o & & & & 6 \\
\hline & & & Toma de riesgo & & $\mathrm{O}$ & & & & 6 \\
\hline & & & $\begin{array}{l}\text { Esfuerzo por sobresalir } \\
\text { Flexible Dinámico }\end{array}$ & & $\begin{array}{l}0 \\
0\end{array}$ & & & & $\frac{6}{1}$ \\
\hline & & \multirow{4}{*}{$\begin{array}{c}\text { Entorn } \\
\text { o }\end{array}$} & $\begin{array}{l}\text { Identificación de } \\
\text { oportunidades }\end{array}$ & 2 & 0 & 0 & o & 0 & 2 \\
\hline & & & $\begin{array}{l}\text { Evaluación y elección de } \\
\text { estas oportunidades }\end{array}$ & \multirow{2}{*}{$\begin{array}{l}2 \\
4\end{array}$} & \multirow{2}{*}{$\begin{array}{l}0 \\
0\end{array}$} & \multirow{2}{*}{$\begin{array}{l}1 \\
2\end{array}$} & \multirow{2}{*}{0} & \multirow{2}{*}{$\begin{array}{l}0 \\
0\end{array}$} & 3 \\
\hline & & & Dinamiza la industria & & & & & & 6 \\
\hline & & & Investiga & & 0 & 0 & 0 & 0 & 3 \\
\hline & \multirow{12}{*}{$\begin{array}{l}\text { Empre } \\
\text { sario }\end{array}$} & \multirow{4}{*}{$\begin{array}{l}\text { Individ } \\
\text { wo }\end{array}$} & $\begin{array}{l}\text { Necesidad de logro } \\
\text { Cree en sus capacidades } \\
\text { Cumple metas y objetivos }\end{array}$ & \multirow{2}{*}{$\begin{array}{l}4 \\
3 \\
2\end{array}$} & \multirow{2}{*}{$\begin{array}{l}0 \\
0 \\
0\end{array}$} & \multirow{2}{*}{$\begin{array}{l}0 \\
0 \\
3\end{array}$} & \multirow{2}{*}{$\begin{array}{l}0 \\
0 \\
0\end{array}$} & \multirow{2}{*}{$\begin{array}{l}0 \\
0 \\
0 \\
0\end{array}$} & $\frac{2}{4}$ \\
\hline & & & Es innovador & & & & & & $\frac{3}{5}$ \\
\hline & & & $\begin{array}{l}\text { Impulsa la creación de } \\
\text { una organización, busca } \\
\text { el beneficio }\end{array}$ & \multirow{2}{*}{$\frac{2}{2}$} & 0 & \multirow{2}{*}{$\begin{array}{l}0 \\
2 \\
0\end{array}$} & \multirow{2}{*}{$\begin{array}{l}0 \\
0 \\
0\end{array}$} & & 2 \\
\hline & & & Busca el crecimiento & & O & & & O & $\frac{4}{2}$ \\
\hline & & & $\begin{array}{l}\text { Conocimiento del } \\
\text { contexto }\end{array}$ & 2 & 0 & 1 & ? & 0 & 5 \\
\hline & & Entorn & $\begin{array}{l}\text { contexto } \\
\text { Sostenibilidad }\end{array}$ & $\begin{array}{l}4 \\
0\end{array}$ & o & 2 & 0 & o & 2 \\
\hline & & 0 & Competitividad & 0 & 0 & 1 & 0 & 0 & 1 \\
\hline & & & Maneio de información & 2 & 0 & o & o & O & 2 \\
\hline & & & Capacidad de gestión & 2 & 0 & 0 & 0 & o & 2 \\
\hline & & & $\begin{array}{l}\text { Establecimiento de redes } \\
\text { en cadenas productivas }\end{array}$ & 0 & o & 1 & 0 & 0 & 1 \\
\hline & & Proces & $\begin{array}{l}\text { Reconocimiento en el } \\
\text { medio para generar } \\
\text { sostenibilidad }\end{array}$ & 2 & 0 & 0 & 0 & 0 & 2 \\
\hline & & & $\begin{array}{l}\text { Identifica características } \\
\text { de las personas con las } \\
\text { ouales trabaja, se }\end{array}$ & 7 & Q & 0 & 0 & 0 & 1 \\
\hline
\end{tabular}

Fuente: Elaboración Propia -Analisis Manifiesto-

Se identificaron de acuerdo a los tipos de empresarios las caracteristicas y codigos -planteados desde la perspectiva teorica- más recurrentes en las entrevistas -exploratorias, semies-estructuras y estructuradas- que permitieron inductivamente desarrollar el analisis descriptivo y la consideración de nuevos codigos emergentes necesarios para dar respuesta al proposito de nuestro estudio.

Esquema 1. Factores Críticos u codigos emergentes

Fuente: Elaboración Propia -Analisis Latente

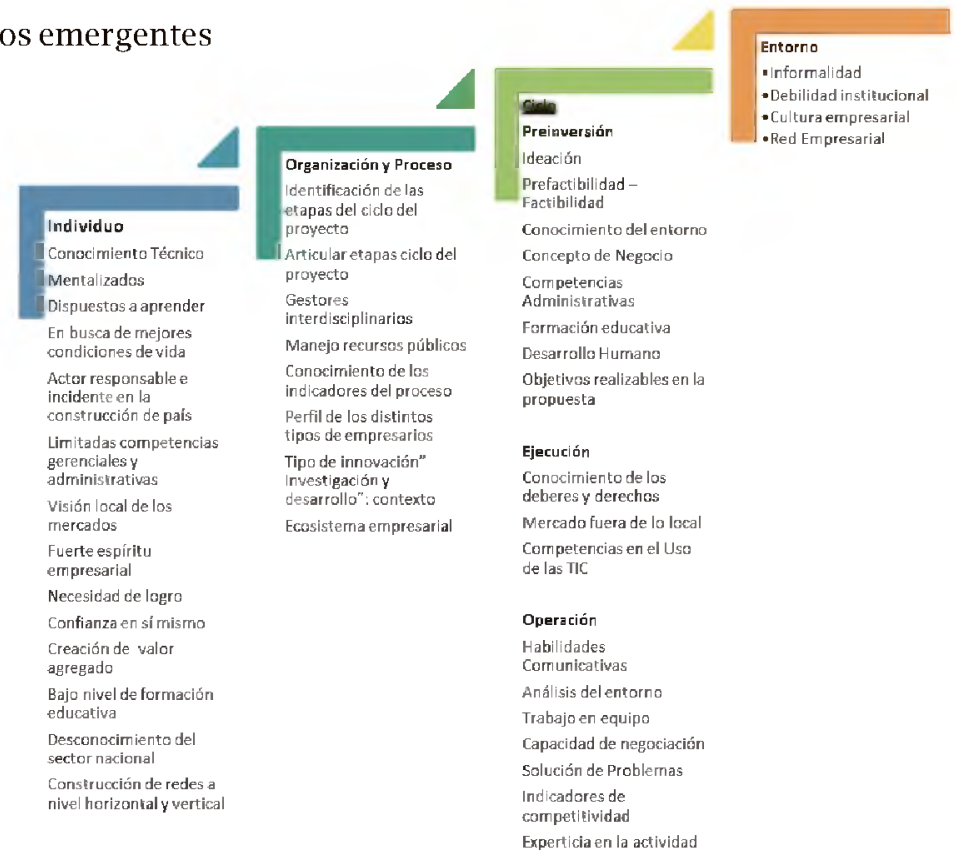




\section{Hallazgos y Conclusiones}

En concordancia con la metodología, revisión documental en fuentes secundarias y análisis de los datos cualitativos, expuestos en los hallazgos encontrados, fue posible dar respuesta a los cuatro objetivos específicos que permiten conocer el objetivo general de la investigación.

1. Perfil de los Emprendedores y origen de la idea de negocio de las iniciativas beneficiadas con recursos del Fondo Emprender.

\section{Individuo}

- Para el caso de los emprendedores del Huila financiados por el Fondo Emprender, la investigación realizada permite caracterizarlos como personas reconocidas por su experiencia y conocimiento técnico previo en la actividad que da origen a su propuesta, mentalizados, dispuestos a aprender y consolidar su iniciativa empresarial, por lo que esta representa una opción para mejorar su calidad de vida. Los empresarios buscan a partir de su emprendimiento generar beneficios directos relacionados con el incremento de sus ingresos y como una oportunidad alternativa atractiva de empleo de beneficio personal y familiar.

La gran debilidad de este actor son sus limitadas competencias gerenciales y administrativas, que en los casos de éxito es suplida parcialmente con el soporte de un grupo interdisciplinario que trabaja con él. Se caracteriza por sus ganas de salir adelante y aunque predomina una visión local de mercados, tiene conciencia como actor responsable en la construcción de país y percibe el trabajo como una condición básica de dignificación del ser humano.

"La paz de un país se busca (...) generando empleo, y que la gente este ocupada y de esta manera poder quitarnos muchos malos pensamientos y muchos Colombianos dejarán de pensar mal para hacerle daño a los demás". (Empresario participante)

\section{Características Individuo}

- A los emprendedores participantes los caracteriza su fuerte espíritu empresarial, necesidad de logro, confianza en sí mismo, que los promueve en avanzar. Tales características, parecen fundamentarse en la necesidad de "salir adelante", alcanzar una mejor calidad para el futuro de sus familias.

- Los emprendedores buscan crear valor a partir de sus actividades, realmente sienten y creen que la iniciativa empresarial es el camino que permitirá mejorar sus condiciones de vida. No obstante, si bien los emprendedores poseen un fuerte espíritu empresarial, manifiesto como el deseo y ganas de salir adelante, estas variables se ven limitadas, generalmente, por la debilidad en su bajo nivel de formación educativa y el desconocimiento del sector al cual se adscribe el proyecto en el contexto regional, nacional e internacional.
- En lo referente a las formas organizacionales que se conforman para el desarrollo de las iniciativas empresariales, el componente de innovación y diferenciación se circunscribe al contexto donde se pretende desarrollar la iniciativa, el cual se caracteriza por:

Oportunidades de mercado locales para sus productos y servicios.

Dificultades de los emprendedores para adaptarse a los cambios de regulación, normatividad.

En la dimensión del proceso de desarrollo de la iniciativa empresarial, la construcción de redes a nivel horizontal -lazos empresariales- son débiles, y no se evidencia una integración hacia agentes u organizaciones de la cadena de valor del emprendimiento y la innovación, desde las cuales se logre aprender e interiorizar mejoras. Los empresarios ven su proceso, una oportunidad para mejorar sus condiciones de vida, pero no lo ven -inmediatamente- como una ocasión para acumular dinero.

El entorno como dimensión que incide en el éxito o fracaso de un emprendimiento es considerado por los emprendedores entrevistados de suma importancia. De acuerdo a su visión, el entorno se caracteriza por el predominio de mercados locales para la comercialización de sus productos, las autoridades municipales adolecen de políticas que faciliten las decisiones de los emprendedores como por ejemplo, las relacionadas con la ubicación de la empresa y los emprendedores tienen el convencimiento de que su iniciativa tiene un impacto significativo en la sociedad.

2. Factores críticos y lecciones de aprendizaje de las iniciativas Empresariales financiadas con recursos del Fondo Emprender

\section{Dimensiones: Organización y Proceso}

Como resultado del trabajo de investigación es posible extraer importantes lecciones -factores críticos- de aprendizaje, que facilitan la aplicación de acciones de mejoramiento en cada una de las etapas del proyecto y en los procesos de asesoría y acompañamiento:

\section{Relacionadas con el ciclo del proyecto}

1. Resulta fundamental en el proceso de desarrollo de un proyecto de emprendimiento, que desde un principio los emprendedores identifiquen las etapas del ciclo del proyecto y las características necesarias en cada una de ellas: pre-inversión, inversión (ejecución) y operación. En esa medida, comprender que cada etapa tiene relación con la siguiente y requiere de competencias específicas y complementarias que determinan en buena parte el éxito del proyecto.

2. Conocer el ciclo del proyecto debe llevar al emprendedor a identifican conceptualmente las etapas en su desarrollo, pero no necesariamente 
implica que sea un proceso continuo en el tiempo, por varios motivos: relacionados con procesos administrativos, de carácter presupuestal que los emprendedores suelen desconocer y que generan en el proceso, desmotivaciones, incertidumbre $y$ retrasos en los plazos previstos.

3. Necesaria la participación de gestores (facilitadores) con competencias en gerencia de proyectos y capacidad de comprender que entre una etapa del ciclo del proyecto y la siguiente, es razonable que se requieran ajustes en aspectos como: a) Cambio en el valor de las cotizaciones, b) restricciones presupuestales, c) modificaciones en la normatividad, d) cambio de acuerdos preestablecidos con un proveedor, e) retraso de un proveedor, f) demoras en los giros de los recursos. g) surgimiento de nuevos competidores, cambio en la tasa de cambio, desarrollo de nuevas tecnologías, entre otros.

4. Los emprendedores suelen desconocer la dinámica de los proyectos que son financiados con recursos públicos, en donde según la naturaleza y origen de los recursos, es indispensable acatar ciertas condiciones y normatividad.

5. Cada etapa del ciclo del proyecto genera unos entregables verificables que deben ser previstos por el equipo -emprendedor, gestor e interventor-. En ese orden de ideas, el rol de un gestor (facilitador) es lograr que los emprendedores diseñen e implementen el conjunto de actividades que permitan cumplir con éxito el alcance de cada una de las etapas del ciclo del proyecto.

6. Para el caso del Huila, llama la atención el predominio de emprendimientos individuales, es decir, son relativamente escasos los emprendimientos realizados por equipos de trabajo. Este puede ser un tema de interés para futuras investigaciones de tal manera que se pueda comparar con lo que sucede en otras regiones del país y en los que se analice del conjunto de proyectos presentados y aprobados cuántos tienen el componente de innovación, qué tipo de mercados se atienden y que opciones tienen no solo de sobrevivir, sino de crecer y lograr un desarrollo integral (mercados, tecnología, financiero, talento humano).

7. Aunque al SENA atiende en emprendimiento diferentes tipos de población: técnicos, tecnólogos de la Institución, universitarios, población desplazada, jóvenes rurales, es igualmente válido para cualquier institución pública o privada que preste servicios similares, tener en cuenta indicadores de línea base de los emprendedores, pues según las características y competencias iniciales de cada población objetivo, hay que optar por estrategias diferenciadas que permitan una mejor eficiencia y eficacia en los procesos de asesoría y acompañamiento en cada una de las etapas del ciclo del proyecto, así como del tipo de innovación que se pretende desarrollar.
8. En materia de innovación, para el caso del Huila, aún predominan los emprendimientos surgidos por necesidad, caracterizados por mantener condiciones de sobrevivencia, atención a mercados locales, bajo nivel de complejidad, limitada capacidad de crecimiento, rentabilidad baja y escasa relación con proyectos de investigación; en tal sentido, si bien constituyen una alternativa laboral, presentan dificultades para generar nuevos puestos de trabajo estables y bien remunerados.

9. La articulación y cohesión institucional juega un papel fundamental en la construcción de una cultura de la investigación, que reconozca la importancia de la educación, la investigación, la innovación y el emprendimiento de alto impacto. Aunque se reconoce la importancia de atender la demanda de servicios de población vulnerable para la creación de emprendimientos por necesidad, también hay que trabajar simultáneamente en el desarrollo de emprendimientos basados en el conocimiento y esta labor solo se puede materializar cuando se fortalezca la relación - EstadoAcademia - Sector Productivo-.

\section{Relacionadas con la etapa de pre-inversión}

10. En la etapa de pre-inversión en Colombia hizo carrera que los emprendedores van de la idea directamente al plan de negocio, práctica que ha generado problemas, especialmente relacionados con la débil estructuración del proyecto. La experiencia deja en evidencia la importancia de trabajar con mayor énfasis en el proceso de identificación de la idea (ideación) y el análisis con mayor rigurosidad de los componentes claves de un proyecto (factibilidad): análisis del mercado, estudio técnico (localización, tamaño e ingeniería), análisis legal y organizativo, cuantificación de las inversión, presupuesto de ingresos, costos y gastos, evaluación financiera e impacto social.

11. La presión por cumplir con indicadores numéricos (metas), en la práctica, incide en que el gestor (facilitador), termina siendo copartícipe (coautor) en el proceso de elaboración el plan de negocio, con el riesgo, de que el emprendedor pierda empoderamiento en su proyecto y presente falencias e inseguridad en las etapas siguientes del ciclo del proyecto.

12. En relación con la ideación - proceso es fundamental para que los emprendedores filtren con argumentos su propuesta y se apropien y "enamoren" de su idea-, el análisis encontrado permite inferir que para el caso del Huila, muchos emprendedores desconocen las características del sector del cual hace parte de la idea, no tienen lo suficientemente conceptualizado en que consiste el negocio y no han hecho cuentas del tamaño de la oportunidad de un mercado objetivo; en tal sentido, lo primero que planteamos es que una 
vez identificada la idea, es necesario tamizarla teniendo en cuenta tres elementos básicos: 1) estudiar el comportamiento y tendencias del sector en el cual se circunscribe la idea (ejemplo: educación, salud, energía, vivienda, etc), 2) hacer una primera aproximación al concepto de negocio, entendido como la síntesis de lo que el emprendedor considera como elementos claves para que la empresa funcione correctamente y 3) determinar y valorar (cuantificación) la oportunidad.

13. Una de las debilidades de mayor frecuencia en los planes de negocio evaluados por FONADE, es la relacionada con la calidad de las investigaciones de mercado. Es importante, cualificar estos análisis incluyendo el estudio del comportamiento del sector al cual pertenece el proyecto, así como evidenciar la aceptación del producto o servicio en el mercado, mediante pruebas de validación. Estas prácticas permiten disminuir el nivel de incertidumbre y tomar decisiones con información más confiable.

14. Los empresarios participantes de las iniciativas huilenses presentadas en las convocatorias del Fondo Emprender, tienden a sobreestimar los escenarios de mercados en la presentación de sus planes de negocio, así como en el planteamiento de los objetivos e indicadores a cumplir los cuales se pueden catalogar como "muy optimistas". En parte esta situación se explica por el desconocimiento de las características y tendencias del sector al cual pertenece el proyecto y la falta de mayor rigurosidad en la investigación de mercados.

15. Es crucial los limitantes que presentan los emprendedores a nivel formativo en algunos casos y en el uso de las TICs. Estos dos elementos -uso de las TICs y el acceso a internet- constituyen una limitación y condicionan el trabajo de los emprendedores en el Departamento, caracterizados por incluir también jóvenes rurales, de baja formación académica y ubicados en zonas agrarias en donde el acceso a la señal es inestable.

16. Se encuentra necesaria, de acuerdo a la población participante, disponer de un mayor número de gestores para los procesos de asesoría y acompañamiento.

"el inconveniente que se presenta en si es que la unidad de emprendimiento no tiene bastantes gestores y entonces se quedan cortos en el tema" (Emprendedor 1).

"el problema como le digo del SENA, es el poco personal para tantas cosas que ellos tienen que hacer, sería bueno que le inyectaran más personal" (Emprendedor 2)

"digamos que si el SENA en sí, que empleara más gestores, más apoyo operativo, para que ellos tuvieran más oportunidad de apoyarnos, porque entereza ellos se la meten toda, por decir algo ellos se trasnochan, madrugan, se quitan tiempo de su familia y todo el cuento para poderle ayudar a los emprendedores" (Emprendedor 3)

17. La interdisciplinariedad y transdisciplinariedad en la composición de los equipos de gestores en las unidades de emprendimiento -Neiva, Garzón, Pitalito, La Plata- son una fortaleza que hacen del SENA, la entidad con mejor capacidad de respuesta a la demanda de servicios de asesoría y acompañamiento en el Huila.

18. En el planteamiento de las actividades programadas a realizar en la fase de ejecución y relacionadas en el Plan Operativo, los tiempos no corresponden a los requeridos para adelantar las labores requeridas. Esto significa que no necesariamente existe la coherencia suficiente entre el trabajo previo del emprendedor y la información utilizada en el aplicativo financiero.

19. Los emprendimientos se pueden clasificar en dos grandes categorías, aquellos que surgen por necesidad los cuales están asociados a población con escaso nivel de formación y corresponden por lo general a iniciativas de población vulnerable y aquellos que están relacionados con la aplicación del conocimiento.

\section{Relacionadas con la etapa de ejecución}

20. El desconocimiento de requisitos exigidos por la entidad encargada de aprobar los giros correspondientes a la etapa de inversión, tales como características que debe reunir una factura de compra, requisitos de las cotizaciones, preparación de informes para las entidades encargadas del seguimiento, debilidades en los procesos de sustentación de informes de avance y registros inadecuados son factores que con frecuencia influyen en retrasos en el giro de los recursos, creando situaciones de incertidumbre en los emprendedores y en algunos casos de desmotivación.

21. Si bien se reconoce capacidad técnica y experiencia en relación con las propuestas de los emprendedores, es necesario incluir en los procesos formativos una visión que supere lo local y el desarrollo de competencias administrativas $y$ gerenciales por su impacto tanto en las etapas de ejecución como de operación del proyecto.

22. Imperante resulta contar con un mayor conocimiento en los requisitos, obligaciones legales, cambios normativos y del sector al cual pertenece el proyecto para la sostenibilidad y crecimiento empresarial en el mediano y largo plazo. 
23. Se reconoce la existencia de iniciativas emprendedoras atractivas, pero se presentan casos de debilidades en lo relacionado con la definición de una adecuada segmentación y canales de comercialización apropiados.

24. En ese sentido, resulta pertinente que el emprendedor asuma una mayor autonomía, compromiso, visión más amplia de la iniciativa empresarial, asimile y aplique las sugerencias y/o criticas, pero que finalmente desarrolle una mayor capacidad de toma de decisiones como líder y responsable de su emprendimiento.

25. El trabajo predominantemente individual de los proyectos de emprendimiento en el Huila, limita la posibilidad de complementariedad de conocimientos que puede aportar el trabajo en equipo y mejor aún si se trata de interdisciplinariedad. La experiencia indica mayor probabilidad de éxito si se complementa el conocimiento técnico con el conocimiento de mercados, administrativo y financiero.

\section{Relacionadas con la etapa de operación}

26. En el Huila en los proyectos financiados por el Fondo Emprender, predominan aquellos relacionados con el sector agropecuario, especialmente de café y ganadería, indicador en parte de la vocación regional, pero también de la necesidad de incluir en los procesos formativos nuevas ofertas de nivel técnico, tecnológico y profesional en nuevas áreas asociadas al uso de tecnologías de la información y la comunicación, diseño de software, inteligencia competitiva, gerencia de proyectos que incluso permitan mejorar los procesos de producción tradicionales.

\section{Dimensión: Entorno}

En relación con esta dimensión sustentaremos tres reflexiones:

27. Legalidad de los Empresarios beneficiarios del capital semilla vs la informalidad en el entorno: Este escenario que genera riesgo en los emprendedores por necesidad, es el relacionado con el crecimiento de la informalidad que no tiene que cumplir obligaciones de ley y se constituye en un competidor directo o indirecto, que vende a menores precios, no tienen costos fijos y no tienen controles de calidad.

\section{Dificultades del esquema institucional}

Es visible la limitada cohesión institucional de las instituciones que hacen parte de la cadena de valor del emprendimiento, la ausencia de una política pública adecuada en los municipios, la inexistencia o falta de regulación en los planes de ordenamiento territorial, entrega de permisos, formalización legal, entre otros. Factores que exponen a los emprendedores a situaciones no previstas y generan incertidumbre y retrasos en el proceso de implementación de sus iniciativas empresariales.

29. Débil cultura y red empresarial en el departamento. Baja cohesión en el establecimiento de una red empresarial visible, fuerte, en la que intervengan las universidades, instituciones públicas y los empresarios, en ese orden de ideas, es necesario que se reconozca que la que la investigación básica y aplicada genera oportunidades de innovación y emprendimiento de alto impacto regional y nacional.

\section{Número de Iniciativas Empresariales financiadas con recursos del Fondo Emprender}

De acuerdo al informe ejecutivo del SENA Fondo Emprender acerca del Programa de Emprendimiento, desde el origen del Fondo al año 2012 se realizaron 110 convocatorias de las cuales 28 tuvieron una cobertura nacional y 82 regionales con recursos del capital semilla del orden de $\$ 186.270,3$ millones de pesos. En total se crearon 3.035 empresas con un potencial de generación de empleo de 16.000 plazas en 558 municipios de 31 departamentos del país (Fondo Emprender, 2013).

De acuerdo a registros consultados en la página web de Fondo Emprender, los datos acumulados desde el año 2003 a 2014 en el Huila se han creado 390 empresas con un potencial en generación de empleo 3.176 plazas en 33 municipios beneficiados y con recursos aprobados de $\$ 26.944,5$ millones de pesos, destacándose como el departamento líder en gestión de recursos del Fondo Emprender con una participación del orden del $9,4 \%$ recursos totales asignados $(\$ 284.741,5$ millones de pesos) (Fondo Emprender, 2015).

El departamento del Huila en concordancia con la información estadística a diciembre de 2013, suministrada por la Dirección de Emprendimiento del SENANeiva, se han beneficiado con recursos del capital semilla 238 jóvenes rurales, 38 aprendices SENA, 31 universitarios y 32 emprendedores en general (SENANeiva, 2015).

Gráfica 1. Población Beneficiaria Fondo Emprender en el Huila período 2003-2013

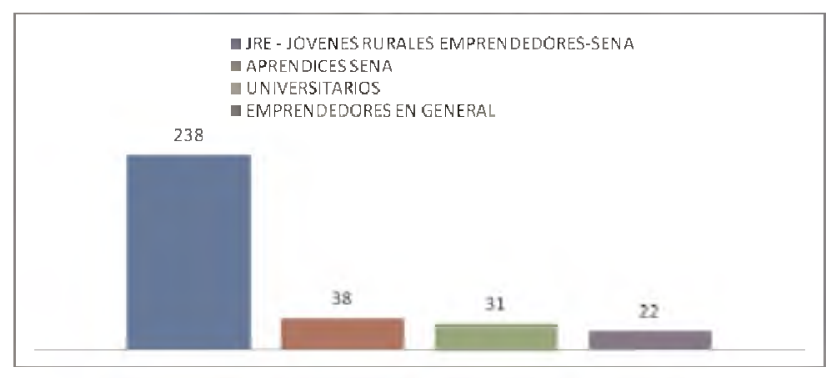

Fuente: Unidad de Emprendimiento Neiva, Servicio Nacional de Aprendizaje Sena. Información estadística suministrada directamente a los investigadores (2015). 
Gráfica 2. Número de planes de negocio por productos en el Huila. Perído2003-2014

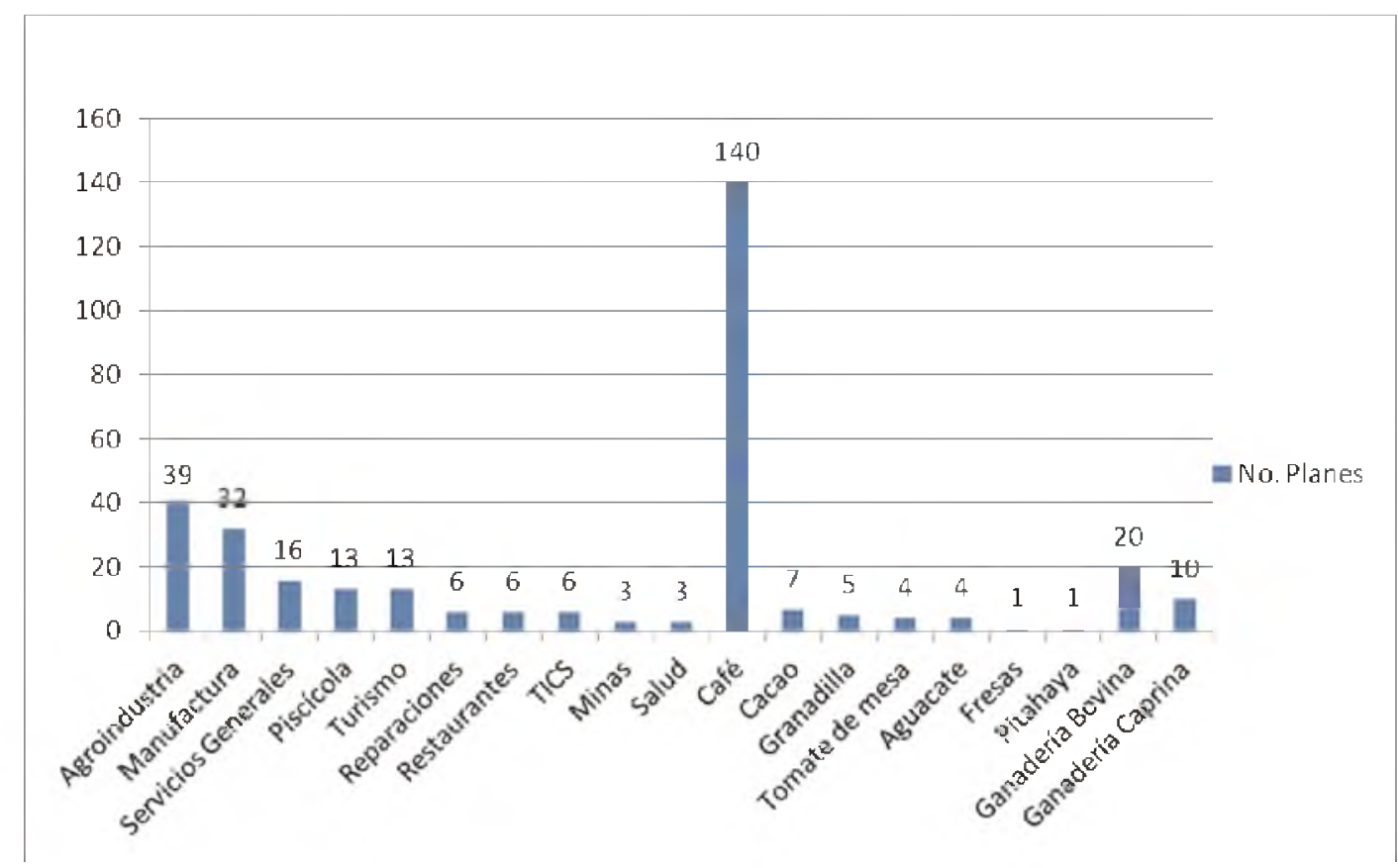

Fuente: Unidad de Emprendimiento Neiva, Servicio Nacional de Aprendizaje Sena. Información estadística suministrada directamente a los investigadores (2015).

La información relacionada permite deducir el posicionamiento y tendencia creciente de recursos asignados y administrados por el Fondo Emprender y la gran participación que en los recursos asignados las iniciativas presentadas por los jóvenes rurales, destacándose en el sector agropecuario el café y la ganadería.

Tabla 3. FONDO EMPRENDER-Resultados Convocatorias Nacionales y Regionales HUILA- Resumen-2002-013

\begin{tabular}{|c|c|c|c|c|c|c|c|}
\hline & & $\begin{array}{l}\text { Recursos } \\
\text { Ofertados } \\
\text { A nivel } \\
\text { nacional } \\
\text { Smillones }\end{array}$ & $\begin{array}{c}\text { Planes } \\
\text { presentados } \\
\text { A niwel } \\
\text { regional }\end{array}$ & $\begin{array}{c}\text { Planes } \\
\text { Aprobados } \\
\text { Huila }\end{array}$ & $\begin{array}{l}\text { Recursos } \\
\text { asignados } \\
\text { al Huila }\end{array}$ & $\begin{array}{c}\text { No. } \\
\text { Empleos } \\
\text { directos } \\
\text { generados }\end{array}$ & $\begin{array}{l}\text { Ingresos } \\
\text { proyectados } \\
\text { porventas } \\
1^{\circ} \text { año }\end{array}$ \\
\hline TOTALES & Wacional & $\$ 249.274$ & 950 & 279 & $\$ 16.646$ & 1395 & $\$ 25.506$ \\
\hline TOTALES & Regional & $\$ 5.687$ & 150 & 50 & $\$ 3.423$ & 257 & $\$ 5.712$ \\
\hline $\begin{array}{l}\text { Gran total } \\
\text { Fondo } \\
\text { Emprender }\end{array}$ & & $\$ 254.961$ & 1.100 & 329 & $\$ 20.069$ & 1652 & $\$ 31.218$ \\
\hline
\end{tabular}

Fuente: Unidad de Emprendimiento Neiva, Servicio Nacional de Aprendizaje Sena. Información estadística suministrada directamente a los investigadores (2015)

\section{Ultimas consideraciones y nuevas indagaciones investigativas:}

- Es indispensable en el corto plazo actualizar los instrumentos y guías utilizados tradicionalmente utilizados por el Fondo Emprender, dado que hay nuevas herramientas que se pueden asimilar para definir el concepto de negocio, como el modelo planteado en el libro "Business Model Generation" por Alex Osterwalder. Es importante que en las primeras etapas, el equipo emprendedor pueda expresar y mejor si lo hace de manera gráfica, los factores o requisitos que hacen posible que la idea propuesta funcione adecuadamente. 
- Dentro del grupo de emprendimientos exitosos estudiados -aquellos emprendimientos empresariales que aprobaron el año de interventoría y recibieron condonación-, es destacable la conformación de un grupo de apoyo administrativo del SENA, encargado de asesorar directamente a los empresarios en temáticas administrativas, contables, comerciales, prospectivas. Con respecto a los emprendedores que no fueron exitosos, se recomienda no perder la experiencia en estos casos y hacer un seguimiento y acompañamiento para futuras iniciativas. Por lo general empresarios exitosos, no lo fueron en un principio, pero aprovecharon las experiencias negativas en oportunidades de aprendizaje.

- Se recomienda para las empresas financiadas por el Fondo Emprender que están en la etapa de operación establecer indicadores complementarios que permitan identificar cuáles escasamente sobreviven, cuáles tienen posibilidades de crecer y desarrollarse integralmente, cuántas atienden mercados locales y cuántas han llegado a otros mercados, cuáles mantienen condiciones de innovación, cuántas han crecido en generación de nuevos empleo, cuántas han crecido en patrimonio. Este conjunto de indicadores, facilitarán determinar con mayor certidumbre el impacto en el sector y en la región de las empresas creadas.

- Si las empresas puestas en operación se asocian a un organismo vivo, en el cual su sobrevivencia y desarrollo depende de la capacidad de adaptarse a los cambios del entorno, un tema de futuras investigaciones es dar respuesta a la pregunta ¿Cuáles son las causas que hacen que una vez creadas las empresas financiadas con recursos del Fondo Emprender, muchas desaparecen rápidamente?.

- Un significativo número de instituciones públicas y privadas se han hecho partícipes de la cadena de valor del emprendimiento; sin embargo, para el caso colombiano, vale la pena indagar sobre las razones por las cuales unas regiones presentan diferencias significativas con respecto a otras en la consolidación de una cultura del emprendimiento y la innovación. Identificar con claridad esas razones, es otro tema para nuevas investigaciones que puede facilitar la definición de estrategias que ayuden a superar las acciones institucionales individuales y lograr un mayor impacto en el desarrollo regional.

\section{Referencias bibliográficas}

Atlas ti. (2009). Brochure. Obtenido de http://atlasti.com/wp-content /uploads/2014/07/atlas.ti6_brochure_2009_es.pdf

Banco de la Republica . (s.f.). Obtenido de http://www.banrep.gov.co /docum/ftp/borra388.pdf

Benzaquen, J., Del Carpio, L. A., Zegarra, L. A., \& Valdivia, C. A. (2010). Un indice regional de competitividad para un país. Revista Cepal 201, 69-86.

Cardona, M., Dinora, V., \& Tabares, J. (2012). Las Dimensiones del emprendimiento empresarial: La experiencia de los prograas Cultura E y Fondo Emprender en Medellín. Cuadernos de Investigación Eafit, 1-64.
Castro, M. E. (2010). El estudio de caso como metodología de investigación y su importancia en la dirección y administración de empresas. Revista Nacional de Administración de Empresas, 3154.

Definición De. (3 de 10 de 2014). Obtenido de Definición D: http://definicion.de/plan-de-negocios/

Emprendimiento Empresarial . (20 de 04 de 2010). Cadena de Valor. Obtenido de Blog: http://miempresa2010.blogspot.com/2010 /04/cadena-de-valor.html

Emprendimiento Empresarial 2010. (20 de 04 de 2010). Cadena de Valor. Obtenido de Blog: http://miempresa2010.blogspot.com /2010/04/cadena-de-valor.html

Escobar, W. (22 de 09 de 2014). Mprende. Obtenido de Mprende: http://mprende.co/legal/algunos-antecedentes-pol\%C3\%ADticoecon\%C3\%B3micos-de-la-ley-de-emprendimiento-en-colombia-0

Escobar, W. (22 de 09 de 2014). Mprende. Obtenido de http://mprende. co/legal/algunos-antecedentes-pol\%C3\%ADtico-econ\%C3\%B3 micos-de-la-ley-de-emprendimiento-en-colombia-0

Esquivias, M. T. (2004). Creatividad: Definiciones, Antecedentes y Aportaciones. Revista Digital Universitaria UNAM, 2-17.

EUSKADI+INNOVA. (3 de 10 de 2014). ¿Qué es Innovación tecnológica? . Obtenido de EUSKADINNOVA: http://www.euskadinnova.net/es innovacion-tecnologica/ambitos-actuacion/innovaciontecnologica/162.aspx

Fondo Emprender. (2014). Convocatorias Fondo Emprender. Obtenido de http://nuevo.fondoemprender.com/SitePages/Convocatorias .aspx

Fondo Emprender. (s.f.). Marco Normativo. Obtenido de http://nuevo. fondoemprender.com/SitePages/Normatividad.aspx

Fondo Emprender. (2013). Estadísticas físico recibidas a Unidad de Emprendimiento de Neiva-SENA.

Fondo Emprender. (2015). Resultados de Impacto. Recuperado de HYPERLINK "http://www.fondoemprender.com/SitePages/ Indicadores.aspx" $\backslash \mathrm{t}$ "_blank" http://www.fondoemprender.com/ SitePages/Indicadores.aspx

Formichella, M. M. (2004). Fl Concepto de Emprendimiento y su relación con la educación, el empleo y el desarrollo local. Recuperado en: ftp:/ftp.unicauca.edu.co/Facultades/FIET/ Materias/Gestion_tecnologica/2005/Clase\%2012/Nuevos/ emprendydesarrollolocal.pdf.

Gartner, W. (1985). Conceptual Framework for Describing the Phenomenon of New Venture. The Academy of Management Review, 696-706.

Gartner, W. B. (1989). Who is an entrepreneur?is the wrong question. Entrepreneurship, Theory and Practice. Summer, $47-68$.

Global Entrepreneurship Monitor Colombia (20-03-2015). Colombia Global Entreprenurship Monitor 2011-2012. Disponible en http://gemcolombia.org/publications/gem-colombia-2011/

Maya, M. (2001). Una introducción a los métodos cualitativos: Modúlo de entrenamiento para Estudiantes y profesionales. Obtenido de http://www.ualberta.ca/ iiqm//pdfs/introduccion.pdf

Ministerio de Comercio, I. y. (07 de 2009). Ministerio de Comercio, Industria y Turismo. Obtenido de: http://www.mincit.gov.co/ minindustria/descargar.php?id=24353

Muñoz., J. (2014). Innovación en la pyme para aumentar la competitividad. Disponible:

http://www.pyme.go.cr/expopyme2014/docs/presentaciones 14 /InnovacionPymeAumentarCompetitividad.pdf

Orrego, Clara Ines. (2012). Emprendimiento y Formación de la Voluntad: Una aproximación Fenomenológica . Tesis Doctoral. Medellín: EAFIT.

Ruiz, A. (s.f.). Triangulación (Metodología). Obtenido de ECURED: http://www.ecured.cu/index.php/Triangulacl\%C3\%B3n_(Metodol $0 \mathrm{\%} \% \mathrm{C} 3 \mathrm{ADa})$

SENA. (s.f.). Acuerdo N 0004 de 2009 . Obtenido de: http://mgiportal.sena.edu.co/downloads/2009/juridica/ acuerdo-0004-de-2009.pdf

Sistema Electrónico de Contratación Pública. (2014). Sistema Electrónico de Contratación Pública. Obtenido de: https://www. contratos.gov.co/consultas/detalleProceso.do?numConstancia $=14-4-2382011$

Tarapuez, E., Guzman, B., \& Parra, R. (Mayo, 2014). Orígenes y componentes de la Politica de Emprendimiento en Colombia 2002-2010. En II Encuentro Nacional de Grupos de Investigación en Historia Empresarial y Emprendimiento. Popayan, Colombia. 